\title{
La revisión de los grados universitarios: valoración de la convergencia europea del Grado en Publicidad y Relaciones Públicas en España
}

\section{Revision of university degrees: evaluation of the European convergence in Advertising and Public Relations degrees in Spain}

Sonia López Berna. Universidad de Alicante (sonia.lopez@ua.es)

Natalia Papí Gálvez. Universidad de Alicante (natalia.p@ua.es)

Marta Martín Llaguno. Universidad de Alicante (marta.martin@ua.es)

Recibido el: 15/01/16 - Aceptado el: 06/10/16

\section{Resumen:}

Este estudio sintetiza las valoraciones de informantes clave sobre la repercusión de la convergencia europea en la formación en Publicidad y Relaciones Públicas en España. Se recogen, mediante entrevistas enfocadas, las opiniones de académicos sobre la elaboración de los planes de estudio; las modificaciones en la oferta educativa; la relación entre la universidad y el ámbito empresarial o el interés de la academia por el estudio de la profesión. Los resultados destacan la especificidad del grado frente a la generalidad de la licenciatura como el cambio más significativo. La selección de las asignaturas en las universidades públicas ha estado condicionada por la plantilla de profesorado; la convergencia no ha alentado el estudio de los perfiles profesionales y la relación entre la universidad y sector profesional es aún escasa.

Palabras clave:

Educación superior; publicidad; relaciones públicas; convergencia europea; perfiles profesionales

\section{Abstract:}

This study summarizes key informants' assessments of the repercussions of the European convergence in Advertising and Public Relations degrees in Spain. Academics'opinions about the development of degree curricula are gathered through focused interviews; noting changes to educational offerings; the relationship between universities and the business sector or the interest of the academy in the study of the profession.

The results highlight the specificity of the degree as opposed to the generality of the bachelor degree as the most significant change. The selection of subjects at public universities has been conditioned by teaching staff. The convergence process has not encouraged the study of professional profiles and the relationship between the university and the professional sector is still inadequate.

Key words:

Higher education; advertising; public relations; European convergence; professional profiles 


\section{Introducción}

\subsection{Los estudios de Publicidad y Relaciones Públicas en España}

Transcurridos cuatro cursos académicos desde la culminación de la convergencia al Espacio Europeo de Educación superior, en el año 2010, las universidades españolas se hallan en pleno proceso de revisión de los grados universitarios. Es el momento idóneo para valorar la forma en la que se desarrolló este cambio, y conocer el impacto real que ha tenido sobre la comunidad universitaria.

Pero antes de abordar el objeto de estudio que nos ocupa, es necesario resumir en pocas líneas la evolución de los estudios en Publicidad y Relaciones Públicas en España, desde su origen hasta la finalización del proceso de convergencia, para apreciar las diferencias más significativas derivadas del cambio.

Los antecedentes a los actuales estudios en Publicidad y Relaciones Públicas en España podrían ser datados en las décadas de los años 20 y 30. Los primeros pasos de la disciplina en el siglo XX tienen como protagonista a Pedro Prat Gaballí, quien en 1915 y 1917 ejerce labor docente en la Cámara de Comercio y Navegación de Barcelona impartiendo las materias, "Una nueva técnica: La Publicidad Científica” y "Ventas por correspondencia y Campañas de Publicidad”. Prat Gaballí tuvo también un papel decisivo en el desarrollo de las asociaciones profesionales. En 1922 funda junto con un grupo de profesionales dedicados a la enseñanza de la técnica publicitaria la Asociación de profesionales de la Publicidad. Ésta se disolvió dando paso al Publi-Club, Asociación de estudios de Publicidad y Organización. Publi-Club participó en Congresos Internacionales de Publicidad y organizó en 1934 el Congreso Internacional de la Unión Continental de Publicidad (Pérez Ruiz, 2001: 91 y ss.). La ciudad de Barcelona se convierte en la cuna de la Publicidad desde el punto de vista formativo, lo que favoreció la aparición y proliferación de asociaciones profesionales (i.e. Méndiz Noguero, 2000; López-Berna, 2007).

La Guerra Civil española interrumpe bruscamente la trayectoria formativa iniciada en la disciplina publicitaria, y no es hasta el año 1964, fecha en la que se promulga el "Estatuto de la Publicidad" (ley 61/1964, de 11 de junio), cuando se retoma esta dinámica. Este estatuto legal define por primera vez en España la normativa de actuación profesional e impone la creación de una institución destinada a la formación de los publicitarios: el Instituto Oficial de la Publicidad. Esta institución asume en los años sesenta algunos de los objetivos encomendados en la actualidad tanto a la universidad como a los colegios profesionales, tales como la investigación en materia publicitaria, la difusión de información, la orientación para los profesionales y la enseñanza de la Publicidad. Comienza en esta época a impartirse en la Escuela Oficial de Publicidad las enseñanzas que conducen al título de Técnico publicitario. Esta Escuela aporta el primer plan de estudios relacionado con esta disciplina, aunque todavía dista mucho de la estructura de contenidos que tendrán los planes de estudios universitarios (Fernández Poyatos, 2006). 
Los estudios de comunicación en España adquieren el rango de universitarios en el año 1971 con la creación de las Facultades de Ciencias de la Información en la Universidad Complutense de Madrid, la Autónoma de Barcelona y la Universidad de Navarra. Más tarde, concretamente en 1991, el Ministerio de Educación y Ciencia establece por Decreto las líneas de los nuevos planes de estudio, convirtiendo las tres ramas de la licenciatura en Ciencias de la Información en tres carreras distintas: Periodismo, Publicidad y Relaciones Públicas y Comunicación Audiovisual (ANECA, 2004: 100).

Una vez en la Universidad, la licenciatura de Publicidad y Relaciones Públicas vive una primera etapa de asentamiento (1971-89) en la que se imparten programas derivados de la Escuela Oficial de Publicidad. En el curso 1986-87 se configura todos los planes de estudio, hasta ese momento muy dispares ente sí, en materias troncales, obligatorias y optativas, según el Real Decreto 1497/1987. En 1989 comienza la proliferación de licenciaturas que se prolonga hasta el año 2000. A partir de 1991, se renuevan los planes de estudio pasando de 5 a 4 años en la mayoría de los casos; la organización temporal se articula por cuatrimestres, lo que supone la duplicación del número de asignaturas; y se renuevan contenidos (Méndiz Noguero, 2000: 211 y ss.).

Ya en el año 2010, en España había un total de 236 campus/sedes universitarios, de los cuales 154 corresponden a universidades públicas, 69 a universidades privadas, además de las 13 sedes con las que cuentan la UIMP y la UNED (MEC, 2010: 5). Según los datos del INE para el curso 2009-2010 era posible cursar estudios de Publicidad y Relaciones Públicas en 32 universidades.

\subsection{La convergencia al Espacio Europeo de Educación Superior}

En España, la convergencia al EEES se articuló desde el Estado a través del Ministerio de Educación, Cultura y Deporte (MECD) y la ANECA.

A tal efecto, el Ministerio publicó en 2003 el documento-marco que sienta las bases de la actual estructura de dos niveles: grado y postgrado. Según el texto, el nivel de Grado daría lugar a la obtención de un título con cualificación profesional en el mercado laboral europeo, y el nivel de Postgrado, para cuyo acceso es necesario haber superado el primero, desemboca en el título de máster y/o doctorado. El propósito es que el estudiante adquiera conocimientos generales en el Grado que se complementen con materias específicas en el Postgrado (García-García, 2010: 67).

Con respecto a los objetivos formativos de Grado, el documento-marco deja patente la orientación profesional de este primer nivel. Los estudios universitarios deben orientarse a competencias que capaciten al graduado al ejercicio de la profesión. El Proyecto Tunning (2000) constituye un marco de referencia para comprender este nuevo enfoque, que se apoya, a su vez, en experiencias reales tales como los Proyectos de redes temáticas Sócrates-Erasmus y los proyectos piloto ECTS (Aboites, 2010; Universia). 
Para ello, se considera "esencial en el proceso de diseño y elaboración de las enseñanzas oficiales del nivel de Grado no sólo su armonización con las titulaciones consolidadas en otros países europeos en cada uno de los ámbitos científicos, técnicos y artísticos, sino la estrecha colaboración entre los responsables académicos y los de las asociaciones y Colegios Profesionales" (MECD, 2003: 8). Este documento refleja la necesidad de involucrar de manera directa al sector profesional en el proceso de Bolonia y de reducir distancias, en definitiva, entre el ámbito profesional y el académico (Mérida-Serrano, 2006).

La ANECA es el organismo que mayor protagonismo cobró en la adaptación de la formación reglada al EEES en España. Puso en marcha un proceso que se tradujo, a efectos prácticos, en tres convocatorias de ayudas económicas que promovían el trabajo en red de las universidades españolas, con el propósito último de elaborar un libro blanco de referencia. En las convocatorias se presentaron algunas pautas, con pretensiones de obligado cumplimento, para ordenar y hacer comparables los trabajos de las distintas redes. Los libros blancos se realizaron con el objeto de identificar las destrezas profesionales sobre las que, a posteriori, se construirían los planes de estudio de las titulaciones (ANECA, 2003: 12). Los perfiles profesionales se convierten, así, en la piedra angular sobre la que se vertebran los nuevos planes de estudio.

\subsection{La orientación a competencias profesionales de los estudios de Grado: el caso de Publicidad y RR.PP.}

Tal y como sostiene Riesco González (2008: 81), el EEES “se apunta al movimiento de la empleabilidad”, lo que implica que los títulos deben preparar a los futuros egresados para el acceso al ejercicio profesional. Partiendo de esta premisa, los planes de estudio se articulan en torno a competencias profesionales, o lo que es lo mismo, a "capacidades efectivas para llevar a cabo exitosamente una actividad laboral plenamente identificada".

Los planes de estudio deben centrarse en la adquisición de competencias por parte de los estudiantes, "ampliando por tanto (aunque no excluyendo) el tradicional enfoque basado principalmente en contenidos y horas lectivas". Se deberá hacer énfasis en los métodos de aprendizaje de dichas competencias y en los procedimientos para evaluar su adquisición. "Esta referencia tanto a competencias como a conocimientos es necesaria para facilitar la movilidad de los titulados universitarios dentro de la Unión Europea (...)” (MEC, 2006: 6).

En educación superior se debe considerar "la interrelación de los saberes y su integración - mapa de competencias -" (Tejada Fernández y Ruiz Bueno, 2016: 22). Una competencia profesional, en muchas ocasiones, no puede adquirirse/desarrollarse en una sola materia o curso, sino que se debe abordar desde distintas asignaturas y niveles, teniendo en cuenta los diferentes grados de dominio. La evaluación de las competencias transversales condiciona la configuración de los planes de estudio de los Grados, ya que los contenidos de las materias deben estar interconectados entre sí y en ningún caso deben solaparse. Además, la formación en competencias profesionales comporta partir de situaciones y problemas reales, con diseños curriculares organizados en prácticas: "el aprendizaje de competencias es 
siempre funcional; su vinculación con el contexto y la necesidad de la acción implica planteamientos metodológicos y organizativos abiertos, múltiples, variados y flexibles" (Tejada Fernández y Ruiz Bueno, 2016: 25).

En este contexto, las universidades iniciaron el proceso de adaptación al EEES con, al menos, dos fuentes de documentación primarias: el documento marco publicado por el Ministerio en el 2003 y los libros blancos, y con una directriz clara: que los Grados fueran una realidad en el curso académico 2009-2010.

En el caso de la Publicidad y las RR.PP., con el propósito de identificar los perfiles profesionales, se elaboró el Libro Blanco de los títulos de Grado en Comunicación (ANECA, 2004), sustentado sobre una demanda profesional diferenciada para cada una de las titulaciones. El planteamiento del documento difirió del mantenido por la Comisión de evaluación del proyecto “Títulos de Grado en Comunicación”. Tras la revisión del documento, los evaluadores expusieron en su informe que no se justificaba la necesidad de tres títulos diferentes. No obstante, recomendaron la edición del Libro Blanco y su trámite al Ministerio y al Consejo de Coordinación Universitaria (ANECA, 2004: 13).

En el Libro Blanco se recogen 4 perfiles profesionales (ANECA, 2004: 33): Director/a de comunicación, investigador/a y consultor/a estratégico en Publicidad y RR.PP.; Investigadores/as, Planificadores/as y Compradores de medios; Creativo/a y diseñador/a y Gestor/a de comunicación corporativa. En cambio, algunos autores (Castellblanque, 2006; Lanas, 2011; Zurita, 2011) han hecho saber que este documento de referencia no incluye todos los perfiles profesionales más importantes. De hecho, el convenio colectivo estatal suscrito para las empresas de Publicidad (BOE, febrero 2010) recoge hasta un total de 41 perfiles profesionales.

El ámbito de la comunicación se caracteriza por ser cambiante, aparecen y se consolidan nuevos perfiles profesionales con cierta asiduidad. No en vano, el mismo año que el proceso de convergencia culmina, 2010, y se ponen en marcha muchos de los Grados de Publicidad verificados por la ANECA, el 80\% de las agencias encuestadas por Corredor-Lamas y FarfánMontero (2010) habían modificado su estructura, añadiendo divisiones especializadas encargadas de coordinar las actividades online con incorporaciones de nuevos perfiles, tales como: Directores de mobile marketing, expertos en redes sociales o directores creativos digitales. Actualmente, las agencias de medios más importantes ofrecen más servicios por efecto de los nuevos medios (Flores-Vivar, 2009; Papí-Gálvez, 2015). Aún así, los Grados son la oferta educativa que en mayor medida parece incluir en sus contenidos perfiles vinculados a Internet, incluso frente a los Postgrados, lo que muestra, por una parte, la influencia de la orientación profesional en la reforma universitaria (Papí-Gálvez y López-Berna, 2012) y, por otra, la contradicción de este enfoque con la adquisición de conocimientos generales en el Grado que se desprende del documento-marco del MECD (2003).

Esta realidad parece dar la razón a algunas críticas a las bondades del enfoque y al procedimiento habilitado para su aplicación. Antes, los planes de estudio consistían en una relación de disciplinas identificadas a través de descriptores 
generales que permitía la planificación de los contenidos por áreas de conocimiento. Ahora, a la desaparición de la llamada troncalidad se le une la habilitación de un proceso abierto en cuanto a operativa se refiere.

En consecuencia, y pese a disponer de un libro blanco de referencia, los contenidos pueden quedar condicionados, en última instancia, por elementos contextuales a la labor del propio profesor, tales como la "organización educativa, la cultura profesional (...), la propia estructura departamental (...) las normas de cada institución, los valores, (...)” -y desde luego"las tradiciones" (Mérida-Serrano, 2006: 5). Por ello, tanto estos procesos como el peso que la profesión tuviera en el origen de la titulación pueden repercutir en el éxito de la orientación profesional de los estudios universitarios de ciertas especialidades (García-García, 2010; García, Domínguez y Mayor, 2011).

El papel de la ANECA en la fase de formación de los nuevos planes de estudios es limitado, pues su objetivo es evaluar las propuestas de las universidades en el marco del EEES y no diseñar el título. Por tanto, es necesario centrar el análisis en las propias universidades y, en concreto, en aquellas personas que coordinaron la gestación de los Grados.

Según lo expuesto, el objetivo principal de este estudio es sintetizar las opiniones y valoraciones de informantes clave acerca de las repercusiones de la convergencia europea en la formación reglada en Publicidad y las Relaciones Públicas en España. En especial, se pretende explicar:

1) El proceso de elaboración de los planes de estudio de los grados;

2) Las modificaciones en la oferta educativa;

3) La relación entre la universidad y el ámbito empresarial -los vínculos institucionales-,

4) El interés académico por el estudio de la profesión.

\section{Metodología}

Para responder a los objetivos nos servimos de la técnica de la entrevista en profundidad enfocada individual a partir de "casos ideal-típicos o casos guía". La entrevista en profundidad es una "técnica para obtener información mediante una conversación profesional con una o varias personas para un estudio analítico de investigación o para contribuir en los diagnósticos o tratamientos sociales (...)". De este modo, la entrevista "toma la forma de relato de un suceso narrado por la misma persona que lo ha experimentado, y desde su punto de vista; es en sí el contexto en el cual se elabora este relato, y crea una situación social para que pueda tener lugar; y en ese relato el entrevistador desempeña el papel de facilitador o transmisor" (Ruiz Olabuénaga, Aristegui y Melgosa, 2002: 76). El término "entrevista enfocada” fue acuñado por Robert Merton (1946) para describir una variedad del tipo general de entrevistas no dirigidas. "Es un tipo de entrevista que siempre trata de un núcleo o foco de interés (...) va dirigida a un individuo en concreto, caracterizado y señalado previamente 
por haber tomado parte en esa situación o haber vivido esa experiencia". Las características que mejor definen una entrevista enfocada son (Ruiz Olabuénaga, Aristegui y Melgosa, 2002: 77; Vallés, 2002: 20):

- La persona entrevistada es un sujeto de quien se sabe que ha intervenido en una situación particular.

- $\quad$ El entrevistador conoce de antemano, directa o indirectamente, esta situación y la ha analizado sistemáticamente. En base a este análisis, desarrolla una guía de entrevista en la que se señalan los puntos de mayor interés para la investigación y la hipótesis que confieren mayor relevancia a los datos que se van a recoger.

- La entrevista se concentra en la experiencia subjetiva de los sujetos entrevistados con el objeto de conseguir de ellos su definición de la situación. -La entrevista está siempre abierta a que respuestas o relatos imprevistos den pie a nuevas hipótesis e interpretaciones de la experiencia.

La selección de los casos guía puede definirse como "un procedimiento en el que el investigador idea el perfil del caso mejor, más eficaz y más deseable de una población y, posteriormente, encuentra un caso del mundo real que se ajusta a aquél de forma óptima” (Rodríguez Gómez, Gil Pérez, García Jiménez, 1999: 137).

El perfil del informante que puede dar respuestas a las cuestiones nucleares relacionadas con los resultados de la aplicación del EEES a los estudios de Publicidad y Relaciones Públicas integra a:

1) Académicos e investigadores que pertenezcan a una selección de universidades españolas (públicas y privadas) en las que se imparta el Grado en Publicidad y Relaciones Públicas.

2) Que ocupen o hayan ocupado un cargo académico durante el proceso de adaptación de las licenciaturas a los grados que les faculte para la participación directa en dicho proceso, esto es, para la dirección, coordinación, supervisión y/o ejecución del mismo (Vicedecanos de titulación, Coordinadores o Directores de Licenciatura / Grado en Publicidad y Relaciones Públicas o Vicedecanos de Ordenación Académica).

3) Que dispongan de información relativa a los planes de estudio de los títulos a extinguir (licenciaturas en Publicidad y Relaciones Públicas). Deberán, por tanto, haber formado parte del PDI de la institución académica antes de que el proceso de adaptación a los grados culminara, curso 2009-2010.

4) Que ejerzan o hubieran ejercido como docentes en la Licenciatura y/o en el Grado en Publicidad y Relaciones Públicas.

El paso previo a la selección de los entrevistados fue determinar las universidades del estudio. Así, para la selección de dichas universidades se procuró, en primer lugar, que hubiera la misma representación de universidades públicas que de privadas. Después, utilizando los datos del INE referentes al curso 2009/2010, se seleccionaron, de entre todas las universidades en las que se oferta la titulación de Publicidad y Relaciones Públicas en España, aquellas con un mayor número 
de alumnos matriculados. Finalmente incluimos en el estudio 8 universidades (las cuatro públicas con mayor número de alumnos de entre todas las públicas y las cuatro privadas con mayor número de alumnos de entre todas las privadas) que suman 7.847 estudiantes matriculados, cifra que supone el 53,53\% de todos los matriculados en España (14.659 estudiantes). Lo que nos permite afirmar que sólo estas 8 instituciones estaban formando en ese momento a más de la mitad de los futuros profesionales en Publicidad y Relaciones Públicas.

Una vez seleccionadas las 8 universidades, se contactó a través del correo electrónico con los informantes clave que se muestran en el cuadro 1.

Cuadro 1. Informantes clave.

\begin{tabular}{|c|c|c|c|}
\hline Universidad & Informante & $\begin{array}{l}\text { Año de } \\
\text { ingreso }\end{array}$ & Cargo \\
\hline ALICANTE & Enric Mira Pastor & 2000 & $\begin{array}{l}\text { Vicedecano de Publicidad y Relaciones Públicas de la Facultad } \\
\text { de Ciencias Económicas y Empresariales }\end{array}$ \\
\hline $\begin{array}{l}\text { CEU CARDENAL } \\
\text { HERRERA }\end{array}$ & $\begin{array}{l}\text { Ma José González } \\
\text { Solaz }\end{array}$ & 1999 & $\begin{array}{l}\text { Vicedecana de Publicidad y Relaciones Públicas de la Facultad } \\
\text { de Humanidades y Ciencias de la Comunicación }\end{array}$ \\
\hline $\begin{array}{l}\text { COMPLUTENSE } \\
\text { DE MADRID }\end{array}$ & $\begin{array}{l}\text { 1. Ma Teresa García } \\
\text { Nieto } \\
\text { 2. Javier Davara }{ }^{1}\end{array}$ & $\begin{array}{l}\text { 1. } 1989 \\
\text { 2. } 1977\end{array}$ & $\begin{array}{l}\text { 1. Vicedecana de Ordenación académica de la Facultad de Cien- } \\
\text { cias de la Información (2005-2009) } \\
\text { 2. Decano de la Facultad de Ciencias de la Información (1998- } \\
\text { 2009) }\end{array}$ \\
\hline $\begin{array}{l}\text { OBERTA DE } \\
\text { CATALUÑA }\end{array}$ & Ferrán Lalueza & 1999 & Director de estudios del Grado en Comunicación \\
\hline RAMÓN LLULL & $\begin{array}{l}\text { Antonio Solanilla } \\
\text { Pascual }\end{array}$ & 1997 & $\begin{array}{l}\text { Director del Grado en Publicidad y Relaciones Públicas. Facultad } \\
\text { de Comunicación. }\end{array}$ \\
\hline $\begin{array}{l}\text { REY JUAN } \\
\text { CARLOS }\end{array}$ & $\begin{array}{l}\text { 1.Esther Martínez } \\
\text { Pastor } \\
\text { 2.Manuel Martínez } \\
\text { Nicolás }^{2}\end{array}$ & $\begin{array}{l}1.2006 \\
2.2005\end{array}$ & $\begin{array}{l}\text { 1. Coordinadora del Grado en Publicidad y Relaciones Públicas } \\
\text { de la Facultad de Ciencias de la Comunicación. } \\
\text { 2. Vicedecano de ordenación académica y de alumnado (2006- } \\
\text { 2008). }\end{array}$ \\
\hline
\end{tabular}

Javier Davara interviene, a petición de la Dra. García Nieto, en calidad de Presidente de la asamblea de Decanos en el año 2004. En ese momento, el profesor Davara desempeñaba el cargo de Decano de la Facultad de Ciencias de la Información de la Universidad Complutense de Madrid.

2 En la parte relativa a la elaboración del grado, dado que la profesora Martínez Pastor aún no desempeñaba su cargo en el año de implementación del mismo, interviene el profesor Manuel Martínez Nicolás, Vicedecano de ordenación académica y de alumnado desde el 2006 al 2008, quien coordinó el proceso de elaboración de los Grados de Periodismo y de Publicidad y Relaciones Públicas. 


\begin{tabular}{|l|l|l|l|}
\hline \multicolumn{1}{|c|}{ Universidad } & \multicolumn{1}{|c|}{ Informante } & $\begin{array}{c}\text { Año de in- } \\
\text { greso }\end{array}$ & \multicolumn{1}{c|}{ Cargo } \\
\hline CEU SAN PABLO & Karen Sanders & 2006 & $\begin{array}{l}\text { Directora del Departamento de Publicidad y comunicación insti- } \\
\text { tucional de la Facultad de Humanidades y Ciencias de la } \\
\text { Comunicación. }\end{array}$ \\
\hline VALLADOLID & $\begin{array}{l}\text { Ma de la Cruz Alva- } \\
\text { rado }\end{array}$ & 2003 & $\begin{array}{l}\text { Vicedecana de Ordenación Académica y de Publicidad y Relacio- } \\
\text { nes Públicas de la Facultad de Ciencias Sociales, Jurídicas y de la } \\
\text { Comunicación. }\end{array}$ \\
\hline
\end{tabular}

Fuente: Elaboración propia.

Las entrevistas se realizaron a lo largo de octubre del 2011. A continuación, se muestra el guión empleado, que fue validado por cuatro profesores, dos de técnicas de investigación social y dos de teoría de la comunicación:

\section{PLANES DE ESTUDIO}

\section{Grados}

- ¿ ¿Qué es para usted un profesional que ejerce la Publicidad y/o las Relaciones Públicas?

- $\quad$ En la actualidad, ¿qué perfiles profesionales en Publicidad y RR.PP. considera que son los más demandados por el mercado de trabajo?

- ¿ ¿Cree que la formación para el ejercicio de los mismos está recogida en el plan de estudios del grado?

- ¿ ¿ ¿Podría decirnos cuál fue el proceso de elaboración del plan de estudios del actual grados en Publicidad y Relaciones Públicas que oferta su Universidad?, -¿Con qué información/datos contaron de partida?, ¿qué competencias tuvieron en cuenta? (Además del libro blanco de títulos de Grados en Comunicación de la ANECA).

- ¿ ¿Qué cambios significativos aprecia entre el plan de estudio de las licenciaturas y el de los grados en cuanto a la formación en competencias profesionales se refiere?

- $\quad$ ¿Se incluyen en el plan de estudios del grado "prácticas en empresas externas"? En caso afirmativo, ¿itienen carácter optativo u obligatorio?

\section{Post-Grados}

- ¿ ¿Qué competencias, destrezas o habilidades profesionales se incluyen en los planes de estudio de la formación post-grados en su Universidad? 
- $\quad i Q u e ́$ diferencia/as esencial/es aprecia entre la formación post-grados y la de grados en cuanto a las competencias/perfiles profesionales se refiere?

\section{ENTORNO PROFESIONAL}

\section{Universidad-empresa}

- ¿ ¿Conoce qué vinculación tiene su Universidad/Facultad con el entorno profesional: Agencias y/o Empresas de Publicidad?

\section{Asociacionismo profesional y Colegiación}

- ¿ ¿Podría decirme qué vinculación tiene su Universidad/Facultad con las asociaciones nacionales o autonómicas pertenecientes al sector publicitario y/o con los Colegios Oficiales de Publicitarios y RR.PP?

\section{INVESTIGACIÓN}

- ¿ ¿Conoce la existencia de proyectos de investigación o convenios de colaboración para la investigación establecidos entre la Universidad/Facultad y empresas u organizaciones del sector publicitario?

- ¿ ¿Conoce la existencia de líneas temáticas, dentro de los grupos de investigación de los diversos departamentos vinculados a los estudios de Publicidad y RR.PP, centradas en el estudio de la profesión del publicitario?

Para contrastar la información proporcionada por los entrevistados, se realizó una recogida de información complementaria a partir del análisis de contenido de las webs de las universidades seleccionadas. Siguiendo un protocolo de codificación se compiló la información disponible sobre la formación postgrado -títulos propios (cursos de especialista, experto y magisters), másteres universitarios y doctorados que tuvieran una relación directa con la Publicidad y las Relaciones Públicas-. Por otra parte, se compilaron los datos relativos a las líneas/grupos de investigación cuyo objeto de estudio fuera la profesión de la Publicidad y/o las Relaciones Públicas.

\section{Resultados}

\subsection{Elaboración del plan de estudios del grado}

Todos los expertos entrevistados coinciden en señalar el Libro Blanco como documento de referencia para elaborar el plan de estudios del grado. La mayoría considera que el trabajo de compilación necesario para la confección de este documento es suficiente. No obstante, se señala la escasez de producción científica centrada en el estudio de la profesión de la Publicidad y/o las Relaciones Públicas. Para paliar esta carencia, los entrevistados apuntan que han recurrido a la infor- 
mación proveniente de las comisiones de trabajo de otras universidades -planes de estudio del grado, memorias elaboradas para la ANECA, etc -, pero en ningún caso han desarrollado estudios de investigación ad hoc o revisiones científicas, tal y como muestra el cuadro 2.

Cuadro 2. Órganos y procedimientos creados para la elaboración del plan de estudios del grado en Publicidad y Relaciones Públicas y fuentes de información consultadas.

\begin{tabular}{|c|c|c|}
\hline Universidad & Comisiones de trabajo & Fuentes consultadas \\
\hline ALICANTE & $\begin{array}{l}\text {-Comisión de titulación con todos los departa- } \\
\text { mentos implicados formada por docentes, } \\
\text { alumnos, egresados, un representante del Colegio } \\
\text { oficial de Publicidad y Relaciones Públicas de la } \\
\text { Comunidad Valenciana y PAS (personal de admi- } \\
\text { nistración y servicios). }\end{array}$ & $\begin{array}{l}\text { Plan de estudios de la Licenciatura y Libro Blanco. } \\
\text { Planes de estudios de las comisiones de otras uni- } \\
\text { versidades: Jaume I y la Complutense de Madrid. } \\
\text { Además, se buscó información sobre las titulacio- } \\
\text { nes de comunicación de algunas universidades } \\
\text { extranjeras, concretamente en EE.UU. }\end{array}$ \\
\hline $\begin{array}{l}\text { CARDENAL } \\
\text { HERRERA } \\
\text { CEU }\end{array}$ & $\begin{array}{l}\text {-Comisiones consultivas formadas por profesorado } \\
\text { de la Facultad, FIAP, Marcel Moliné, y otros profe- } \\
\text { sionales. } \\
\text { - Múltiples reuniones: Se realizaron reuniones de } \\
\text { las tres universidades que componen la Fundación } \\
\text { CEU: Cardenal Herrera CEU, San Pablo CEU y Abat } \\
\text { Oliva CEU. } \\
\text {-Convenios para benchmarking. La Fundación CEU } \\
\text { firmó un convenio con una organización de la Uni- } \\
\text { versidad de Harvard, para formar parte del } \\
\text { Programa Platón. Incluye lo que denominan “con- } \\
\text { trato", el equivalente a las fichas de las asignaturas, } \\
\text { que recoge las competencias y el sistema de evalua- } \\
\text { ción. Es firmado por alumnos y profesores, siendo } \\
\text { de obligado cumplimiento por ambas partes. }\end{array}$ & $\begin{array}{l}\text { Plan de estudios de la Licenciatura y Libro Blanco. } \\
\text { Planes de estudio de algunas universidades de re- } \\
\text { ferencia en EEUU: Harvard entre otras. }\end{array}$ \\
\hline $\begin{array}{l}\text { COMPLU- } \\
\text { TENSE DE } \\
\text { MADRID }\end{array}$ & $\begin{array}{l}\text {-Comisión de Facultad formada por todos los pro- } \\
\text { fesores que impartían docencia en la Licenciatura } \\
\text { en Publicidad y Relaciones Públicas. } \\
\text {-No se contó con el sector profesional. }\end{array}$ & Plan de estudios de la licenciatura y Libro Blanco. \\
\hline
\end{tabular}




\begin{tabular}{|c|c|c|}
\hline Universidad & Comisiones de trabajo & Fuentes consultadas \\
\hline $\begin{array}{l}\text { OBERTA DE } \\
\text { CATALUÑA }\end{array}$ & $\begin{array}{l}\text {-Comisión de titulación con profesores y personal de calidad de } \\
\text { la UOC. } \\
\text {-Entrevistas a profesionales del sector: en agencias de Barce- } \\
\text { lona. }\end{array}$ & $\begin{array}{l}\text { Plan de estudios de la licenciatura y } \\
\text { Libro Blanco. Oferta formativa de al- } \\
\text { gunas universidades de referencia en } \\
\text { Europa, que ofertan también títulos } \\
\text { de carácter más general. }\end{array}$ \\
\hline $\begin{array}{l}\text { RAMÓN } \\
\text { LLULL }\end{array}$ & $\begin{array}{l}\text {-Comisión con todos los profesores de la titulación de Publici- } \\
\text { dad y Relaciones Públicas. } \\
\text {-Consultas informales: Conversaciones puntuales con profesio- } \\
\text { nales con conexión con el centro. No se trató de una consulta } \\
\text { estructurada o formal. No se contó con ninguna asociación } \\
\text { profesional ni Colegio. }\end{array}$ & $\begin{array}{l}\text { Plan de estudios de la Licenciatura y } \\
\text { Libro Blanco. }\end{array}$ \\
\hline $\begin{array}{l}\text { REY JUAN } \\
\text { CARLOS }\end{array}$ & $\begin{array}{l}\text {-Comisión de titulación en la que participaron todos los depar- } \\
\text { tamentos con docencia en los grados. Luego se delegó en } \\
\text { comisiones por titulación formadas por profesores, estudiantes } \\
\text { y Pas. } \\
\text {-Grupos de discusión y entrevistas con profesionales. Pero no } \\
\text { se contó con ninguna asociación profesional ni Colegio. }\end{array}$ & $\begin{array}{l}\text { Plan de estudios de la Licenciatura y } \\
\text { Libro Blanco. }\end{array}$ \\
\hline $\begin{array}{l}\text { SAN PABLO } \\
\text { CEU }\end{array}$ & $\begin{array}{l}\text {-Comisión de Facultad formada por todo el profesorado del } \\
\text { centro. } \\
\text { - Múltiples reuniones: Se realizaron reuniones de las tres uni- } \\
\text { versidades que componen la Fundación CEU: Cardenal Herrera } \\
\text { CEU, San Pablo CEU y Abat Oliva CEU, y se crearon otras com- } \\
\text { petencias vinculadas al CEU. } \\
\text {-No se contó con nadie en representación del sector profesional. }\end{array}$ & $\begin{array}{l}\text { Plan de estudios de la Licenciatura y } \\
\text { Libro Blanco. Planes de estudio de al- } \\
\text { gunas universidades de referencia en } \\
\text { EEUU: Harvard entre otras. }\end{array}$ \\
\hline VALLADOLID & $\begin{array}{l}\text {-Comisión de la sección de Publicidad y Relaciones Públicas } \\
\text { con presencia de todas las áreas con docencia en la licencia- } \\
\text { tura. Ésta delegó en otra comisión de profesores pertenecientes } \\
\text { sólo al área de Publicidad y Relaciones Públicas. } \\
\text {-No hubo representación del sector profesional. }\end{array}$ & $\begin{array}{l}\text { Plan de estudios de la Licenciatura y } \\
\text { Libro Blanco. }\end{array}$ \\
\hline
\end{tabular}

Fuente: Entrevistas a informantes clave

$58 \mid n^{\circ} 23$, pp. 47-71 | doxa.comunicación 


\subsection{Modificaciones en la oferta educativa}

Tres aspectos han sido subrayados por los entrevistados: la alternativa especificidad/generalidad en los planes de estudio y sus condicionantes, la nueva concepción de la enseñanza -programación de contenidos basados en perfiles profesionales- y las características de la formación de los postgrados.

En primer lugar, la oferta educativa ha variado en las universidades estudiadas en virtud de la especificidad/generalidad de los títulos ofertados. Así, la Universidad Complutense de Madrid, la de Valladolid, la Ramón Llull y la CEU San Pablo apuestan por la primera opción, mientras que la UOC y la Rey Juan Carlos lo hacen por la segunda. Dos cuestiones son clave para la toma de decisiones: los perfiles profesionales y los recursos humanos en cada universidad.

Como ejemplo de especificidad, la Universidad Complutense de Madrid, siguiendo la postura de la Conferencia de Decanos, defiende la diferenciación entre los tres títulos, en contra del propósito original del Ministerio que era ofertar un solo grado en comunicación. El argumento que se esgrime, según Javier Davara, Decano de la UCM en ese momento y expresidente de la Conferencia de Decanos, está basado en los perfiles profesionales: "Éstos son muy distintos entre sí, por lo que requieren una formación claramente diferenciada".

En el otro extremo, la UOC, con “vocación generalista”, y donde, según Lalueza, no se plantean titulaciones diferenciadas, pero sí 6 itinerarios sujetos a ciertos perfiles: "Creatividad publicitaria”; "Comunicación corporativa” y "Relaciones Públicas”; “Creación audiovisual”; “Gestión audiovisual”; “Gestión publicitaria” y “Comunicación informativa”. Esta opción responde a la búsqueda de oferta formativa complementaria a la de las universidades públicas, con la intención de captar a un público sutilmente distinto. En palabras del profesor Lalueza: "La UOC no está para competir con las Universidades presenciales sino para complementar su formación. En Cataluña ya hay muchas titulaciones vinculadas a comunicación que son especificas".

En la línea de la UOC está también la Rey Juan Carlos. Así, según el profesor Martínez Nicolás, "esta universidad entendió que los grados en el marco de la estructura de Bolonia tenían que ser generalistas, ya que la especialización venía de la mano de los másteres". Pese a este planteamiento, y en palabras del experto, se elaboró un grado atendiendo a dos áreas de especialización: "la creatividad publicitaria" y la "planificación estratégica".

Martínez Nicolás introduce otro factor explicativo para la selección de asignaturas con vocación más generalista, la fuerza docente: "se tuvo en cuenta, por un lado, los perfiles profesionales incluidos en el Libro Blanco, por otro, con qué fuerza docente contábamos. Y ahí hubo el problema para conectar lo que demanda el mercado con el perfil de los profesores. Hubo que hacer muchas cesiones". Se presume aquí que, para dar cabida en el grado a todo el profesorado estable, se seleccionaron algunas asignaturas cuyo contenido era más genérico, y que no responden a las destrezas profesionales específicas requeridas. 
La naturaleza contractual de los docentes, precisamente, es el punto de origen del argumento del profesor Lalueza de la UOC:

“(...) en nuestra opinión, en el resto de universidades, sobre todo en las públicas dada su estructura funcionarial, el cambio hacia una sola titulación es más difícil, pero en el nuestro era mucho más sencillo, ya que en toda la UOC hay unos 300 profesores a dedicación plena, en cambio hay unos 3.000 colaboradores. Se dedican a trabajar fuera (como los profesores asociados). Si cambias la asignatura, cambias de colaborador. Eso nos da cierta flexibilidad" (Dr. Lalueza- UOC, Barcelona).

La profesora Sanders (Universidad CEU San Pablo) considera que existen diferencias entre el planteamiento de las universidades públicas y privadas sujetas a la estructura laboral de las primeras: “(...) a diferencia de las universidades públicas, donde no se tiene tanto margen de maniobra y se hacen concesiones a asignaturas y a docentes, aquí tenemos más flexibilidad". Sanders menciona aquí la posibilidad de "concesiones" sujetas a la relación contractual y especialidad del docente en las universidades públicas, lo que coincide plenamente con la aportación del profesor Martínez Nicolás, y puede entrar en contradicción con el planteamiento de los grados basados en los perfiles profesionales. De hecho, la consulta externa a profesionales del sector en la elaboración de los grados se reduce en algunos casos a comunicaciones puntuales, según las declaraciones de los expertos.

La profesora Alvarado, de la Universidad de Valladolid, señala con relación a la selección de las asignaturas que se hicieron consultas a través del correo electrónico a determinados profesionales del sector con los que su universidad colabora de manera puntual, “...pero que se trató de consultas de carácter informal”. En este punto añade: "hubo que hacer muchas concesiones entre profesores. Además se trabajó con mucha prisa, con mucha presión, por lo que no se pudo consultar a profesionales tal y como hubiera sido deseable”. Además, con respecto a la orientación de la formación -generalista / específica- y cuando se le pregunta por las diferencias entre la licenciatura y el grado, indica: "La diferencia fundamental es que las materias básicas están más enfocadas hacia la Publicidad. Esto es un poco contradictorio, dado que el espíritu de la convergencia europea se centra en una formación de grado más generalista, y deja la formación específica para los postgrados. Pero nosotros evitamos que las asignaturas fueran tan genéricas como en las licenciaturas, se buscó más especificidad, tanto en las asignaturas obligatorias como en las optativas. Se entendió que así era mejor".

En las universidades públicas, la fuerza laboral y el escaso aporte del sector profesional han podido condicionar el contenido de los grados, y por extensión su orientación generalista/específica.

A modo de resumen, el cuadro 3 muestra, según los entrevistados, aquellas universidades con materias específicas o áreas de especialización en los grados de Publicidad y Relaciones Públicas.

Al margen de la orientación en la oferta educativa, hay cuatro universidades - Alicante, Rey Juan Carlos, CEU Cardenal Herrera y Oberta de Cataluña- que señalan que el cambio más reseñable entre grados y licenciaturas recae en la concep-

60 | n²3, pp. 47-71 | doxa.comunicación 
ción de la enseñanza. La programación de contenidos centrada en las competencias profesionales marca la diferencia. Tal y como afirma el profesor Enric Mira de la UA: “(...) en el grado el esquema de trabajo se basa en la adquisición de competencias profesionales”. Significar aquí la importancia concedida a la realización de prácticas externas. Se trata de una asignatura de carácter obligatorio en todas las universidades, a excepción de dos de ellas: la Complutense de Madrid y la de Alicante. Ambas afirman entender la obligatoriedad de las prácticas como el status ideal, pero en su caso resulta inviable administrativamente dado el elevado número de alumnos.

Cuadro 3. Cambios más significativos en el plan de estudios entre el Grado y la Licenciatura.

\begin{tabular}{|l|l|}
\hline Universidad & Diferencias en el Plan de estudios \\
\hline ALICANTE & $\begin{array}{l}\text { Cambios conceptuales y operativos: Forma de concebir la enseñanza: la licenciatura es una enseñanza } \\
\text { programada de acuerdo con contenidos, y en el grado se basa en la adquisición de competencias profe- } \\
\text { sionales. }\end{array}$ \\
\hline $\begin{array}{l}\text { CARDENAL } \\
\text { HERRERA } \\
\text { CEU }\end{array}$ & $\begin{array}{l}\text { Cambio orientación oferta educativa: En la licenciatura, la formación es generalista, y la especificidad la } \\
\text { marca la optatividad. En el grado, la formación también es generalista, pero la optatividad no es tan } \\
\text { rica, ya que son los másteres los que marcan la especialización. }\end{array}$ \\
\hline $\begin{array}{l}\text { COMPLU- } \\
\text { TENSE DE } \\
\text { MADRID }\end{array}$ & $\begin{array}{l}\text { Cambio orientación oferta educativa: Asignaturas más específicas en el grado que en la licenciatura. } \\
\text { Aumento del número de asignaturas de Relaciones Públicas }\end{array}$ \\
\hline $\begin{array}{l}\text { OBERTA DE } \\
\text { CATALUÑA }\end{array}$ & $\begin{array}{l}\text { Cambio en los sistemas de acceso. La UOC sólo oferta el segundo ciclo de la licenciatura en Publicidad y } \\
\text { el grado no sucede, ya que el alumnado accede en primer curso. } \\
\text { Cambio orientación oferta educativa: Las titulaciones pre-Bolonia tienen modelo basado en conteni- } \\
\text { dos, y más específico, el grado apuesta por una enseñanza más genérica, más transversal. }\end{array}$ \\
\hline $\begin{array}{l}\text { RAMÓN } \\
\text { LLULL }\end{array}$ & $\begin{array}{l}\text { Cambio orientación oferta educativa: } \\
\text { Mayor especificidad y orientación profesional del grado. } \\
\text { Eliminación de los solapamientos entre asignaturas. } \\
\text { Plan de estudios más completo: incluye las denominadas “unidades formativas", que posibilitan la in- } \\
\text { corporación de destrezas profesionales. }\end{array}$ \\
\hline
\end{tabular}




\begin{tabular}{|l|l|}
\hline Universidad & Diferencias en el Plan de estudios \\
\hline & $\begin{array}{l}\text { Cambios operativos: } \\
\text { Positivos: Mayor número de prácticas que en la licenciatura } \\
\text { Regativos: Reducción del número de horas lectivas, y sustitución por horas de trabajo autónomo del } \\
\text { CARLOS }\end{array}$ \\
& $\begin{array}{l}\text { alumno. } \\
\text { Cambios conceptuales: } \\
\text { Positivos: trabajo de fin de grado. } \\
\text { Negativos: Sustitución de asignaturas específicas por asignaturas genéricas. } \\
\text { Incremento del número de asignaturas de Relaciones Publicas en detrimento de las de Publicidad. }\end{array}$ \\
\hline $\begin{array}{l}\text { CEU SAN } \\
\text { PABLO }\end{array}$ & Cambio orientación oferta educativa: Mayor especialización en el grado \\
\hline VALLADOLID & Cambio orientación oferta educativa: Mayor especialización en el grado. \\
\hline
\end{tabular}

Fuente: Entrevistas a informantes clave

Los cambios de orientación de la docencia y los cambios en la oferta educativa son señalados por los entrevistados. Pero, cuando se pregunta a los expertos por los elementos de mejora que estos cambios han supuesto, uno de los aspectos reiteradamente señalado es la planificación de materias centrada en las habilidades profesionales. Una aspiración común entre los entrevistados es que la formación universitaria garantice la capacitación de los alumnos para poder encajar con los perfiles profesionales más demandados. En este sentido, cuatro de las ocho universidades señalan que el mercado busca "Gestores de la comunicación on line"; tres que se requieren "Relaciones Públicas" y "Planificadores estratégicos" y otros tres "Comunicadores todo terreno".

Pese a la preocupación generalizada entre los encuestados porque la oferta educativa permita formar a los alumnos en estas dimensiones, sólo dos universidades de las analizadas afirman recoger específicamente el perfil de "Gestor de la comunicación on line" en los contenidos del grado. Otros cinco centros dicen facilitar parte de la formación necesaria a partir de asignaturas específicas.

Con relación a los otros perfiles profesionales, las competencias propias del perfil de "Relaciones Públicas" y las de "Planificador estratégico" sí se han recogido en los nuevos planes de estudio y su oferta se ha visto incrementada en relación con la licenciatura. En cuanto al perfil del "Comunicador todo terreno", los expertos afirman que tiene su correspondencia en los contenidos del grado, aunque, tal y como muestra la tabla 3, la orientación de los mismos, es más bien específica -y no generalista- , orientada sobre todo hacia la planificación estratégica y la creatividad. 
En el cuadro 4 quedan recogidos los perfiles más demandados por el mercado y su correspondencia en los contenidos del grado.

Cuadro 4. Perfiles profesionales más demandados por el mercado de trabajo y recogidos en el plan de estudios del grado.

\begin{tabular}{|c|c|c|}
\hline Universidad & Perfil más demandado & Recogidos en el grado \\
\hline ALICANTE & $\begin{array}{l}\text { Community manager } \\
\text { Planner en Internet }\end{array}$ & Ninguno de los mencionados. \\
\hline $\begin{array}{l}\text { CEU CARDENAL } \\
\text { HERRERA }\end{array}$ & $\begin{array}{l}\text { Comunicador on line } \\
\text { Ejecutivo de Cuentas } \\
\text { Planificador de medios }\end{array}$ & Todos los mencionados \\
\hline $\begin{array}{l}\text { COMPLUTENSE } \\
\text { DE MADRID }\end{array}$ & $\begin{array}{l}\text { Relaciones Públicas } \\
\text { Comunicador on line }\end{array}$ & Uno de los mencionados: Relaciones Públicas \\
\hline $\begin{array}{l}\text { OBERTA DE CA- } \\
\text { TALUÑA }\end{array}$ & $\begin{array}{l}\text { Profesionales versátiles con conocimientos on } \\
\text { line }\end{array}$ & Todos los mencionados \\
\hline RAMÓN LLULL & $\begin{array}{l}\text { Community manager } \\
\text { Planner } \\
\text { Planificación de medios } \\
\text { Relaciones Públicas } \\
\text { Comunicación en el punto de venta } \\
\end{array}$ & Todos los mencionados \\
\hline $\begin{array}{l}\text { REY JUAN } \\
\text { CARLOS }\end{array}$ & Profesional versátil & Todos los mencionados \\
\hline CEU SAN PABLO & Profesional versátil & Todos los mencionados \\
\hline VALLADOLID & $\begin{array}{l}\text { Responsabilidad social corporativa } \\
\text { Estratega (planner) } \\
\text { Creativo }\end{array}$ & Todos los mencionados \\
\hline
\end{tabular}

Fuente: Entrevistas a informantes clave.

Otro de los cambios en la oferta educativa reside en la posibilidad de que el grado se pueda completar con una oferta de postgrado. No obstante, esta opción no se da siempre. A partir de la revisión las webs de los centros seleccionados, se observa que sólo la mitad de las universidades oferta másteres oficiales, dos ofertan títulos propios; mientras que otras dos no disponen de ninguna formación postgrado vinculada a la Publicidad y las Relaciones Públicas (datos del curso 2010- 
2011). La oferta de postgrado es muy escasa. Los expertos señalan que, debido a su importancia en el proceso de convergencia, esta formación está siendo concebida de manera concienzuda, lo que ralentiza su puesta en marcha. Añaden que la implementación de los grados ha supuesto un esfuerzo que ha relegado a un segundo plano la oferta de postgrado. Es preceptivo plantearse qué ocurrirá con la formación de los graduados en Publicidad y Relaciones Públicas si la oferta postgrado no se ve incrementada de manera notable, ya que, como algunos expertos coinciden en señalar:

“(...) el grado asegura a los estudiantes una empleabilidad, pero con un perfil mucho más general. Los másteres serán los que ayuden a especializar, pero eso es lo que está por ver ya que ¿̇hasta qué punto un graduado, al tener una formación más generalista, y con un año menos, va a ser capaz de dar el mismo resultado en la profesión que un licenciado?, la respuesta es que no va a dar el mismo resultado que un licenciado, porque será necesario hacer ese máster, y la oferta de másteres está todavía por desarrollar" (Dra. González Solaz, Universidad CEU Cardenal Herrera).

\subsection{Vínculos institucionales universidad-sector profesional}

Habida cuenta de la importancia de la orientación profesional y de la docencia por competencias que implica el proceso de Bolonia, una de las cuestiones que queríamos analizar es la situación de los vínculos universidad empresa a partir de los cambios de la convergencia. Todas las universidades estudiadas tienen convenio marco para la realización de prácticas externas, pero sólo dos, la Universidad de Alicante y la Universidad Ramón LLull, tienen además un convenio marco con alguna asociación profesional, firmados ambos antes del proceso de convergencia. Las dos universidades de la Fundación CEU pertenecen a varias asociaciones profesionales, tanto nacionales como internacionales. Este vínculo ya existía antes de que culminara el proceso de convergencia europea, por lo que no guarda relación con el mismo. Esto parece indicar que el cambio de concepción en la enseñanza, centrada ahora en los perfiles profesionales, no ha derivado en una mayor presencia o colaboración del entorno profesional en la universidad, tal y como hubiera cabido esperar.

En lo que respecta a los colegios profesionales, la mitad de las universidades mantiene algún vínculo con ellos: la Universidad de Alicante, la Cardenal Herrera CEU, la Ramón LLull y la Oberta de Cataluña. Este hecho puede ser explicado por la ubicación geográfica de estas instituciones: la Comunidad Valenciana y la Catalana. No en vano dos de los tres Colegios oficiales de Publicitarios y Relaciones Públicas de España tienen competencias en estas dos comunidades.

\subsection{Investigación centrada en la profesión de la Publicidad y las Relaciones Públicas}

Por otra parte, parecía razonable indagar sobre el incremento del interés por el estudio de la profesión a partir de la convergencia europea. La información proporcionada por los entrevistados en este sentido fue muy escasa.

Sólo dos entrevistados afirmaron conocer contribuciones a la investigación desde este ámbito: la Universidad de Alicante 
y la Universidad Ramón Llull. En la Universidad de Alicante, el profesor Mira señala la actividad académica de la profesora Martín Llaguno: "ha realizado investigación directamente vinculada con el sector profesional". Ha trabajado en diversos proyectos con la AEAP (Asociación Española de Agencias de Publicidad) y ha elaborado el Código para la Publicidad Infantil de Juguetes con la AEFJ (Asociación Española de Fabricantes de Juguetes). Además, es miembro del jurado de Autocontrol desde 2007.

En el caso de la Universidad Ramón LLull, la vinculación entre la investigación y la profesión reside en la defensa de una tesis doctoral centrada en el estudio del perfil profesional “ejecutivo de cuentas”, cuya autora es Isabel Solana.

Dada la escasez de información, se optó por realizar una búsqueda adicional en las webs para contrastar los datos. Los resultados confirman el escaso interés en el estudio de la profesión mostrado por los investigadores. En efecto, en la Universidad de Alicante hallamos los dos únicos grupos de investigación que tienen líneas de trabajo centradas en la profesión: Comunicación y sociedad del conocimiento (COSOCO), con la línea de investigación titulada "Profesionalismo y calidad de vida en el trabajo de las industrias culturales en la sociedad del conocimiento”, dirigido por la Dra. Martín Llaguno; y Estudios sobre comunicación estratégica (E-com), con la línea de investigación "Profesionales de la comunicación y la Publicidad”, dirigido por la Dra. Papí Gálvez. Estos grupos existían antes de la convergencia, de modo que, en contra de lo esperado, el proceso de cambio en la educación superior no parece haber alentado la producción científica centrada en la profesión.

Por último, se debe añadir que la Universidad CEU San Pablo ha creado en el año 2011 el denominado Centro de Tendencias de Comunicación, cuyo objetivo es realizar investigación en el ámbito de la Publicidad y las Relaciones Públicas. Tiene varios proyectos de investigación en marcha insertados en las siguientes categorías: "Reputación corporativa on line"; “Comunicación institucional”; “Redes sociales y Publicidad”; “Jóvenes, ética e interactividad” y “Niños y televisión”. Por el momento, ninguno de los proyectos auspiciados por este centro, dependiente de la Facultad de Humanidades de la Universidad CEU San Pablo, tiene como objeto de estudio la profesión, sino más bien los manifiestos publicitarios.

La comunidad científica muestra, así, mayor interés en los efectos de la comunicación que en los procesos que la generan.

\section{Conclusiones}

A la luz de los resultados obtenidos, podríamos iniciar la discusión afirmando que la construcción del EEES para los estudios de Publicidad y Relaciones Públicas en España se ha iniciado desde un mismo punto de partida, el Libro Blanco, pero se ha desarrollado de manera muy dispar, en otras palabras: hemos asistido a una "convergencia divergente".

Los expertos reconocen que la adaptación de las materias no ha sido en ningún caso basada en la evidencia, y que el Libro Blanco condicionó poderosamente la oferta actual. 
Así, en consonancia con el planteamiento sostenido en este documento, ofertar tres grados -uno para cada titulación- en contra del planteamiento mantenido por la Comisión de evaluación del proyecto "Títulos de Grados en Comunicación" (ANECA, 2004: 13), la mitad de los entrevistados destaca la especificidad de los grados frente a la generalidad de las licenciaturas como el cambio más apreciable generado por la reforma.

Sin embargo, la especialidad no se plantea en los grados, sino en la formación postgrado, donde "los objetivos formativos deben ser más específicos y estar orientados hacia una mayor profundización intelectual” (MECD, 2003: 9 y 10). En este contexto, parece pertinente plantearse algunas cuestiones. En primer lugar, si la formación genérica proporcionada a través de los grados, en algunos casos, garantiza la orientación profesional de los egresados. Y si habrá diferencias significativas, en cuanto a acumulo de conocimiento, entre aquellos que reciban formación de grado basada en conocimientos más específicos y aquellos que la reciban más generalista (teniendo en cuenta que, presumiblemente, no todos los graduados tendrán la posibilidad de realizar un máster de especialización). En segundo lugar, hasta qué punto, dada la disonancia entre el planteamiento inicial de la convergencia (formación generalista a través de los grados) y la realidad estudiada (donde se aprecia la formación específica de los grados) se ha logrado en el ámbito de la comunicación el objetivo esencial del proceso de Bolonia: la construcción de un Espacio Europeo de Educación superior común para todos los países, donde los grados posibiliten la obtención de un "título con cualificación profesional en el mercado laboral europeo” (MECD, 2003: 7). Y en tercer lugar, si la formación específica recibida por los estudiantes a través de los grados se adecua a las demandas de un mercado de trabajo que, tal y como afirman los propios entrevistados, demanda profesionales versátiles.

En otro orden de cosas, la selección de asignaturas en la universidad pública y en la privada ha sido desigual. En las primeras, el modelo de plantilla del personal docente e investigador ha sido clave, y la selección de las asignaturas del grado ha podido mantener el status quo de las licenciaturas. La comunidad universitaria deberá valorar de qué manera repercute este hecho en la oferta educativa; dado que ésta podría verse mermada de materias fundamentales para la formación de los estudiantes de grados, y/o recargada de asignaturas cuyos contenidos no son requeridos para la práctica profesional. El cambio en la concepción educativa que ha traído consigo la reforma de los estudios universitarios debe ser entendido como tal en el sentido más estricto del término, solo así podrá ser un cambio de facto (Mérida Serrano, 2006).

Llegados a este punto, es importante subrayar que entre los perfiles más demandados por el mercado en estos momentos está, según los entrevistados, el denominado "todo terreno", en plena concordancia con el espíritu de la homogeneización europea, pero que entra en contradicción directa con el planteamiento de la especificidad de los grados, mantenido de facto por la mayoría de las instituciones. Así, los académicos parecen tener una visión cercana a la realidad del mercado, que coincide con el planteamiento sostenido en el resto de Europa, donde abogan por titulaciones más generalistas (ANECA, 2004: 303). Sin embargo, no hallamos la concordancia en los planes de estudio de los grados. Una 
vez más, el Libro Blanco puede darnos la respuesta. Los autores de este documento mantienen que el planteamiento del resto de países europeos es fruto del escaso avance del proceso de profesionalización de las actividades comunicativas que existe en los mismos (ANECA, 2004: 304).

Siguiendo con los perfiles, y siempre según los entrevistados, otro de los más demandados es el de "Gestor de la comunicación on line”. Este perfil no está recogido en forma de asignaturas específicas, y sólo se alude al medio Internet en algunos programas. Aquí la oferta educativa parece responder más a una inquietud particular que a un criterio común de programación de contenidos. De hecho, en el Libro Blanco sólo se menciona Internet en el perfil de "Creativos/diseñadores". Quizá esto sea porque el Libro Blanco se elaboró en el 2004, y ha sido, sobre todo a partir de esta fecha, cuando se ha producido el espectacular despegue de Internet.

No obstante, y en términos comparativos con la licenciatura, los expertos aprecian un incremento de materias en las que se aborda al entorno on line. Pese a todo, la oferta formativa en torno a este perfil sigue siendo insuficiente. Teniendo en cuenta que la fecha definitiva de implantación de los grados determinada por el Ministerio de Educación fue septiembre de 2010, las universidades podrían haber realizado un trabajo de búsqueda de información complementaria a la proporcionada por el Libro Blanco - sobre todo en lo que a nuevos perfiles profesionales se refiere- o bien haber realizado una actualización de la misma.

Además, y en contra de lo que cabía esperar, el proceso de convergencia no ha alentado la producción científica centrada en el estudio de la profesión. Se hace necesario, por tanto, aumentar el número de trabajos de investigación en este ámbito. La motivación fundamental para la comunidad científica debería hallarse en la necesidad de conocer las nuevas rutinas de trabajo, que derivan en estructuras de agencias no convencionales y en una nueva forma de entender la Publicidad y las Relaciones Públicas. Sólo a través de la investigación de los procesos de trabajo, los perfiles y las competencias profesionales, y/o las formas de asociacionismo, entre otros aspectos, se podrán elaborar planes de estudio orientados hacia el terreno laboral, y basados en la evidencia científica (López-Berna, Papí-Gálvez y Martín-Llaguno, 2011: 229 ).

Con respecto al aporte del sector profesional en la elaboración de los grados, éste ha sido muy escaso.

Sólo dos universidades formaron comisiones de trabajo que incluían a representantes del sector profesional: Alicante y CEU Cardenal Herrera. Ambas implicaron a representantes del Colegio Oficial de Publicitarios de la Comunidad Valenciana. La ubicación geográfica parece ser influencia directa. El hecho de que algunos de los miembros de la junta directiva del colegio sean profesores en estas universidades constituye un elemento facilitador. De hecho, sólo las universidades ubicadas en comunidades que tienen colegios profesionales mantienen algún vínculo con los mismos. Las ubicadas en la provincia de Madrid y en la de Castilla y León no lo mantienen, manifestando, además, que no lo ven conveniente. La academia concede poca importancia a los colegios profesionales y a su aporte a la formación. En contraposición, concede más 
relevancia a las asociaciones profesionales, ligadas a la formación académica en Publicidad y Relaciones públicas desde su origen (Méndiz, 2000: 184).

Continuando con las formas de asociacionismo, las universidades privadas destacan por su vinculación con las asociaciones profesionales. La clave puede estar en la filosofía empresarial de estas instituciones. De facto, algunas de ellas sólo ofertan másteres de especialización en colaboración con empresas privadas.

En cualquier caso, resulta llamativo que sólo dos universidades hayan contado con los profesionales de manera directa a la hora de planificar los grados, teniendo en cuenta que "resultará esencial en el proceso de diseño y elaboración de las enseñanzas oficiales del grado (....) la estrecha colaboración entre los responsables académicos y los de las asociaciones y los colegios profesionales" (MECD, 2003: 7 y 8). La planificación de las asignaturas debe responder a competencias de carácter profesional, por lo que se hace necesario un cambio en la concepción didáctica. Uno de los primeros pasos sería acabar con la escisión existente entre el mundo académico y el laboral (Mérida Serrano, 2006: 10 y 11). En el caso de las universidades privadas, dado que la mayor parte de su profesorado ejerce a su vez fuera de la institución, se puede pensar que el aporte proviene de estos docentes. Sin embargo, en las instituciones públicas esto no sucede. La convergencia requiere un esfuerzo que logre acercar aún más la academia al entorno profesional y las universidades públicas deben ejercer un mayor impulso en este sentido. No obstante, ha sido precisamente la profesionalización de los estudios la que ha suscitado mayor controversia en el proceso de Bolonia. Tildado por los sectores más críticos de "mercantilista", se hablaba de un intento de "privatización de la enseñanza universitaria” (Cazorla González-Serrano, 2011: 15).

A pesar de esto, y a nuestro juicio, se precisa revisar el nexo de unión entre la academia y el sector profesional en general, y el de las universidades públicas en particular. La relación universidad-empresa debería estar centrada en el aporte mutuo de conocimiento, y las entidades privadas no condicionarán, en ningún caso, los programas educativos. Pero la firma de convenios y colaboraciones con asociaciones y colegios es necesaria. Sólo así los contenidos podrán orientarse realmente hacia las competencias profesionales. De esta estrecha colaboración se obtendría un beneficio mutuo: la academia dispondría de información y datos reales que serían claves para asentar los conocimientos teóricos transmitidos en las aulas, y el ejercicio de la profesión quedaría enmarcado por la teoría, lo que contribuiría a reforzar la identidad profesional. Además, es fundamental que los estudiantes conozcan tanto la actividad de las asociaciones profesionales como el papel desempeñado por los colegios.

Después de todo lo expuesto, cabe valorar los planes de estudio tras el curso académico 2013-2014, que trae consigo la primera promoción de grado. Entre otros aspectos, se debe analizar los resultados académicos obtenidos y compararlos con los resultados en las licenciaturas; el porcentaje de graduados que optan por realizar un máster de especialización, tanto en España como en el resto de países de Europa y los tipos de máster; y la inserción de los egresados, tanto de grado como de máster, en el mercado laboral europeo. Además, sería pertinente establecer una comparativa entre España y otros pa- 
íses de Europa para obtener un "mapa descriptivo" de las titulaciones de comunicación en el Espacio Europeo de Educación Superior.

El cambio en la concepción didáctica de la enseñanza universitaria requerido por la convergencia debe ser entendido por todos los agentes implicados, aún hoy, y en plena revisión de los Grados, como la oportunidad de alcanzar la excelencia en la formación de los futuros profesionales en España. No se trata, en ningún caso, de una cuestión baladí, y sería un error obviar que las profesiones son piezas clave en la sociedad y cumplen colectivamente una función social en todos los órdenes (Fernández-Pérez, 2001; De Miguel, 2004).

\section{Referencias bibliográficas}

Aboites, H. (2010): "La Educación Superior latinoamericana y el proceso de Bolonia: De la comercialización a la adopción del proyecto Tuning de competencias”, Educación Superior y Sociedad, n. 15 (1), pp. 25-44.

ANECA (2004): Libro Blanco de los títulos de Grado en Comunicación.

Carr-Saunders, M. A. y Wilson, P. A. (1933): The professions. Oxford: Clarendom Press.

Castellblanque, M. (2006): Perfiles profesionales de Publicidad y otros ámbitos afines. Barcelona: Editorial UOC.

Cazorla González-Serrano, M. C. (2011): “Una aproximación a los aspectos positivos y negativos derivados de la puesta en marcha del Plan Bolonia en la Universidad española”, Revista Jurídica de Investigación e Innovación Educativa, n. 4, pp. 91 -104 .

Corredor Lamas, P. y Farfán Montero, J. (2010): “Demandas y Formación: Nuevos perfiles profesionales para la Publicidad en España”, Pensar la Publicidad, n. 4 (1), pp. 97-116.

De Miguel, A. (2004): Aportación de los Colegios Profesionales a la sociedad. Madrid: Unión Profesional.

Fernández-Pérez, J. (2001): “Elementos que consolidan el concepto de profesión. Notas para su reflexión”, Revista Electrónica de Investigación Educativa, n. 3 (2). [Disponible en: http://redie.uabc.mx/redie/article/view/40/1180] [Consultado el: 09-11-15]

Fernández Poyatos, D. (2006): Orígenes y evolución de la actividad publicitaria en España (1880-1936). Tesis doctoral (inédita), Universidad de Alicante.

Flores-Vivar, J. M. (2009): “Nuevos modelos de comunicación, perfiles y tendencias en las redes sociales”, Comunicar, n. 17 (33), pp. 73-81.

García-García, A. (2010): Espacio Europeo de Educación Superior. Los estudios de comunicación en España. Reflexiones en torno al Libro Blanco. Vivar Zurita, H. (Coord.) Madrid: Icono 14 editorial. 
García, C. M., Domínguez C. Y. y Mayor, C. (2011): "Alacena: repositorio de diseños de aprendizaje para la enseñanza universitaria", Comunicar, n. 19 (37), pp. 37-44.

Grunig, J. y Hunt, T. (2003): Dirección de Relaciones Públicas. Barcelona: Gestión 2000.

Hawes, G. y Corvalán, O. (2005): Construcción de un perfil profesional. Proyecto Mecesup Tal0101. Instituto de Investigación y Desarrollo Educacional. Chile: Universidad de Talca.

Lanas, P. C. (2011): "En clave digital. Nuevo profesionales en publicidad, Telos: cuadernos de comunicación e innovación", n. 87 , pp. $97-100$.

López-Berna, S. (2007): "La profesionalización de la Publicidad: una aproximación desde la Sociología de las profesiones", en Papí Gálvez (eds.). Cuestiones actuales en publicidad y retos para la innovación docente: Los profesionales en el siglo XXI. Alicante: Librería Compás.

López-Berna, S.; Papí-Gálvez, N. y Martín-Llaguno, M. (2011): "Productividad científica en España sobre las profesiones de comunicación entre 1971 y 2009”, Revista Española de Documentación Científica, n. 34 (2), pp. 212-231.

MECD (2003): Documento-marco "La integración del sistema universitario español en el espacio europeo de enseñanza superior". Madrid.

MEC (2010): Documento “Datos y cifras del sistema universitario español. Curso 2010-11”. Madrid.

Méndiz Noguero, A. (2000): “Orígenes, evolución y desafíos actuales de la docencia publicitaria en España”, Comunicación y Sociedad, n. 2, pp. 181-225.

Mérida-Serrano, R. (2006): "Nueva percepción de la identidad profesional del docente universitario ante la convergencia europea”, Revista Electrónica de Investigación Educativa, n. 8 [Disponible en: http://redie.uabc.mx/vol8nol/contenidomerida.html] [Consultado el: 12-10-14]

Merton, R. y Kendall, P. L. (1946): “The focused interview”, American Journal of Sociology, n. 51, 6.

Papí-Gálvez, N. (2015): "Nuevos medios y agencias innovadoras. El caso de las agencias de medios”, El profesional de la información, n. 24 (3), pp. 1699-2407.

Papí-Gálvez, N. y López-Berna, S. (2012): "Medios on line y publicidad. Los perfiles profesionales en Educación Superior”, Vivat Academia, n. 117E, pp. 672-700.

Pérez Ruiz, M. A. (2001): La publicidad en España. Anunciantes, agencias y medios, 1850-1950. Madrid: Fragua.

Riesco González, M. (2008): "El enfoque por competencias en el EEES y sus implicaciones en la enseñanza y el aprendizaje”, Tendencias pedagógicas, n. 13, pp. 79-106.

Rodríguez Gómez, G., Gil Flores, J. y García Jiménez, E. (1999): Metodología de la Investigación cualitativa. Málaga: Ediciones Aljibe. 
Ruiz Olabuénaga, J. L., Aristegui, I. y Melgosa, L. (2002): Cómo elaborar un proyecto de investigación social. Cuadernos monográficos del ICE. Bilbao: Universidad de Deusto.

Tejada Fernández, J. y Ruiz Bueno, C. (2016): “Evaluación de competencias profesionales en Educación Superior: Retos e implicaciones”, Educación XX1, n. 19 (1), pp. 17-38.

Universia España. Portal de universidades españolas. [Disponible en: http://eees.universia.es/europa] [Consultado el: 2209-15].

Vallés, M. S. (2002): Entrevistas cualitativas. Cuadernos metodológicos. Madrid: CIS.

Yañiz, C. (2004): “Convergencia europea de las titulaciones universitarias. El proceso de adaptación: fases y tareas”, Revista de la Red Estatal de Docencia Universitaria, n. 4 (1), pp. 3-14.

Zurita, H. V. (2011): “TIC, Internet y el sector de la comunicación: nuevos perfiles profesionales para una comunicación digital”, Telos: cuadernos de comunicación e innovación, n. 87, pp. 58-62. 